\title{
On the Total-Neighbor-Distinguishing Index by Sums
}

\author{
Monika Pilśniak • Mariusz Woźniak
}

Received: 1 July 2011 / Revised: 9 December 2012 / Published online: 29 December 2013

(C) The Author(s) 2013. This article is published with open access at Springerlink.com

\begin{abstract}
We consider a proper coloring $c$ of edges and vertices in a simple graph and the sum $f(v)$ of colors of all the edges incident to $v$ and the color of a vertex $v$. We say that a coloring $c$ distinguishes adjacent vertices by sums, if every two adjacent vertices have different values of $f$. We conjecture that $\Delta+3$ colors suffice to distinguish adjacent vertices in any simple graph. In this paper we show that this holds for complete graphs, cycles, bipartite graphs, cubic graphs and graphs with maximum degree at most three.
\end{abstract}

Keywords Total proper coloring - Adjacent-vertex-distinguishing index · Neighbor-sum-distinguishing coloring · Total-neighbor-distinguishing index

Mathematics Subject Classification (2000) 05C15

\section{Introduction}

We use Bondy and Murty's book [1] for terminology and notation not defined here. Let $G=(V, E)$ be a simple graph with maximum degree $\Delta=\Delta(G)$. Without loss of generality, we may assume that $G$ is connected. Suppose that $c: V \cup E \rightarrow$ $\{1,2, \cdots, k\}$ is a proper total coloring of $G$. For a vertex $v$, let $f(v)$ denote the total sum of colors of the edges incident to $v$ and the color of $v$. We try to answer the

The research partially supported by the Polish Ministry of Science and Higher Education by a grant N201 $1247 / 33$.

M. Pilśniak $(\varangle) \cdot$ M. Woźniak

Faculty of Discrete Mathematics, AGH University of Science and Technology, al. Mickiewicza 30, 30-059 Krakow, Poland

e-mail: pilsniak@agh.edu.pl 
question, how large $k$ have to be to guarantee that there is a proper coloring of vertices and edges with $k$ colors, so that the function $f$ distinguishes adjacent vertices of $G$. The smallest such $k$ is called the total-neighbor-distinguishing index by sums, and denoted by tndi $\sum(G)$. Complete graphs, cycles, bipartite graphs, cubic graphs and graphs with maximum degree at most three show that $k \leq \Delta+3$ in these cases, but can we always do it with $\Delta+3$ colors?

Conjecure 1 For every graph $G=(V, E)$, the total-neighbor-distinguishing index by sums tndi $\sum(G)$ satisfies the inequality

$$
\operatorname{tndi}_{\sum}(G) \leq \Delta+3
$$

Proper total colorings of simple graphs were considered first by Rosenfeld in [10]. The minimum number of colors among a total proper coloring is called a total chromatic index and denoted by $\chi^{\prime \prime}$. He showed that $\Delta+2$ colors are enough for cliques, for complete bipartite and tripartite graphs, for balanced $k$-partite graphs and for graphs with maximum degree at most three. Next, Kostochka showed the same bound for graphs with maximum degree at most four and five [5], [6]. In the general case the conjecture that $\chi^{\prime \prime}(G) \leq \Delta+2$ is still open.

About 10 years ago, a new trend originated in the topic of graph colorings. Many mathematicians considered colorings (proper, general, total or from lists) such that vertices (all or adjacent) are distinguished either by sets or multisets or sums. In this paper we investigate distinguishing adjacent vertices by sums. Karoński, Łuczak and Thomason in [8] considered general colorings of edges, and they conjectured that three colors are enough to distinguish adjacent vertices by sums. This conjecture is almost proved-Kalkowski showed that five colors are enough [7]. A similar conjecture by Przybyło and Woźniak states that two colors are enough by general total coloring [9]. Recently, some other authors in [3] considered a proper coloring of edges distinguishing adjacent vertices by sums.

Zhang, Chen, Li, Yao, Lu and Wang in [11] investigated a proper total coloring of $G$, but to every vertex $v$ they assigned a set $S(v)$ of colors of the edges incident to $v$ and the color of $v$. By $\chi_{a}^{\prime \prime}(G)$ or by $\chi_{a t}$ they denoted the smallest number $k$ of colors so that there exists a proper total coloring with $k$ colors that distinguishishes adjacent vertices by sets (i.e., $S(u)$ is different from $S(v)$ for every pair of adjacent vertices $u, v)$. We propose to denote this index by $\operatorname{tndi}(G)$ - the total-neighbor-distinguishing index. They considered the cases of cliques, paths, cycles, fans, wheels, stars, complete graphs, complete bipartite graphs and trees. They showed (giving exact bounds for tndi) that $\Delta+3$ colors are enough in these cases and formulated the following conjecture:

Conjecure 2 For every graph $G=(V, E)$, the total-neighbor-distinguishing-index by sets $\operatorname{tndi}(G)$ satisfies the inequality

$$
\operatorname{tndi}(G) \leq \Delta+3
$$

Next, Chen in [2] proved this conjecture for bipartite graphs and for graphs with maximum degree at most three. Hulgan in [4] gave a really short proofs of his results. 
It is easy to observe, that if two vertices are distinguished by sums then they are also distinguished by sets, but not necessarily conversely. In this paper we prove all (except for bipartite graphs) values and bounds of $\operatorname{tndi}_{\Sigma}(G)$, which were shown earlier for $\operatorname{tndi}(G)$.

\section{Results for Several Classes of Graphs}

Observe that a vertex with maximum degree $\Delta$ in $G$ needs exactly $\Delta+1$ colors to color it and all edges incident to it, so a lower bound for the invariant tndi $\sum(G)$ is $\Delta+1$. This is the same as in a proper total coloring of $G$. However, if there exist two adjacent vertices $x$ and $y$ with maximum degree in $G$, then we have to use at least one additional color to obtain different sets $S(x)$ and $S(y)$. This is a very useful observation in proofs in this section.

Observation 1 If a graph $G$ contains two adjacent vertices $x$, y such that $\operatorname{deg}(x)=$ $\operatorname{deg}(y)=\Delta$, then $\operatorname{tndi}_{\Sigma}(G) \geq \Delta+2$.

\subsection{Paths, Cycles, Stars and Complete Graphs}

Proposition 1 Let $P_{n}$ be a path of order $n \geq 3$. Then $\operatorname{tndi} \sum_{\Sigma}\left(P_{n}\right)=4$ for $n \geq 4$, and tndi ${ }_{\Sigma}\left(P_{3}\right)=3$.

Proof Color the consecutive vertices of $P_{n}$ for $n \geq 4$, with $1,2,1,2, \ldots$ and the consecutive edges with $3,4,3,4,3,4, \ldots$. Clearly, this is a proper total coloring distinguishing adjacent vertices by sums, and no such proper total coloring with less colors exists by Observation 1.

Proposition 2 Let $C_{n}$ be a cycle of order $n$. Then $\operatorname{tndi}_{\sum}\left(C_{n}\right)=4$ for $n \geq 4$, and $\operatorname{tndi}_{\Sigma}\left(C_{3}\right)=5$.

Proof If $n$ is even the coloring of $C_{n}$ is the same as the coloring of $P_{n}$. Let $n$ be odd. Color the consecutive vertices of $C_{n}$ with $1,2,1,2, \cdots, 1,2,3$, and the corresponding consecutive edges with $4,3,4,3, \cdots, 4,1,2$. Clearly, this is a proper total coloring distinguishing adjacent vertices by sums, and no proper total coloring with less colors is neighbor sum distinguishing by Observation 1 (see Fig. 1).

Proposition 3 Let $S_{n}$ be a star of size $n \geq 2$. Then $\operatorname{tndi}_{\sum}\left(S_{n}\right)=n+1$.

Proof First we color the edges of $S_{n}$ with colors $1,2, \cdots, \Delta$ and the vertex of maximum degree with the color $\Delta+1$. Next we use color 2 for a vertex incident to the edge with color 1 , and all remaining vertices we can color with 1 . Clearly, this is a proper total coloring distinguishing adjacent vertices by sums, and no such proper total coloring with less colors is possible.

Proposition 4 Let $K_{n}$ be a complete graph of order $n>1$. Then

$$
\operatorname{tndi}{ }_{\Sigma}\left(K_{n}\right)= \begin{cases}n+1, & \text { if } n \text { is even, } \\ n+2, & \text { if } n \text { is odd. }\end{cases}
$$


Fig. 1 A total proper coloring of $C_{2 k+1}$

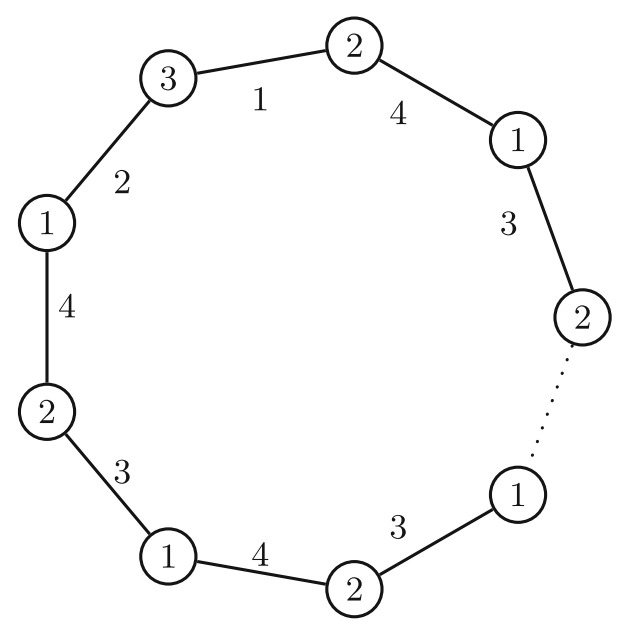

Proof Clearly, no proper total coloring with less than $\Delta+2=n+1$ colors is neighbordistinguishing by sums, by Observation 1 .

First, let $n$ be even. We know from [11] that tndi $\left(K_{n}\right)=n+1$. So there exists a proper total coloring distinguishing adjacent vertices by sets such that for any vertex, exactly one number is missing in its set. Obviously, in every set is missing another color, because the coloring distinguishes by sets, hence sums of colors in all vertices are different.

Next, let $n$ be odd. First we show that tndi ${ }_{\sum}\left(K_{n}\right) \geq \Delta+3$. If there existed a total coloring of $K_{n}$ distinguishing adjacent vertices by sums with $n+1=\Delta+2$ colors, then it would be missing exactly one number (different every once) in every sum. On the other hand, every vertex must have another color, hence we use $n$ colors for vertices. Let $x$ be a vertex with color 1 and let $y$ be a vertex without 1 in its sum. If we consider edges with color 1 , then we see that a matching in color 1 uses $n-3$ vertices $(n-3$ is even), so that there exists a vertex $z \neq y$ in which sum is missing color 1 . Therefore, we must use at least $\Delta+3$ colors in a proper total coloring distinguishing adjacent vertices by sums, if $n$ is odd.

Now, we show that tndi ${ }_{\sum}\left(K_{n}\right) \leq \Delta+3$, if $n$ is odd. We view $K_{n}$ such that its vertices $v_{1}, \cdots, v_{n}$ are situated equidistantly on a circle. In the first step we color all edges incident with $v_{1}$, such that $c\left(v_{1} v_{i}\right)=i$, for $i=2, \cdots, n=\Delta+1$ and a vertex $v_{1}$ with color 1 . Next we consider $v_{2}$ : one edge is colored with 2 , so we put $c\left(v_{2}\right)=3$ and $c\left(v_{2} v_{i}\right)=i+1$, for $i=3, \ldots, n=\Delta+1$ (see Fig. 2).

During the $j$-th step, if $j \leq \Delta+1$, we have colored edges with colors $j, j+$ $1, \cdots, 2 j-2$ [for a color greater than $\Delta+3$ we consider values $\bmod (\Delta+3)$ ]. So we put $c\left(v_{j}\right)=2 j-1,[$ as before, $\bmod (\Delta+3)]$ and $c\left(v_{j} v_{i}\right)=j+i-1 \bmod (\Delta+3)$, for $i=j+1, \ldots, n=\Delta+1$ (see Fig. 3). Notice, $2 n-1=n+2+n-3=$ $\Delta+3+n-3 \equiv n-3$.

This way we obtain a proper total coloring distinguishing all vertices by sums, because the numbers $j-2$ and $j-1$ (where 0 is the color $\Delta+3$ and -1 is the color $\Delta+2)$ are missing in the sum for a vertex $v_{j}$. 


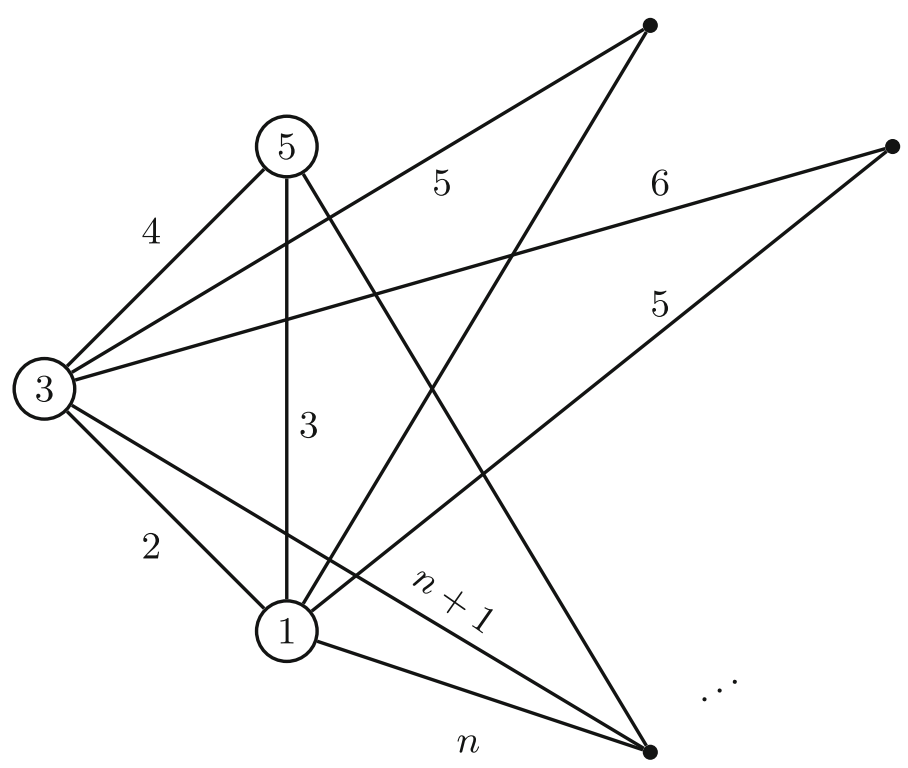

Fig. 2 First two steps of a total proper coloring of $K_{2 k+1}$

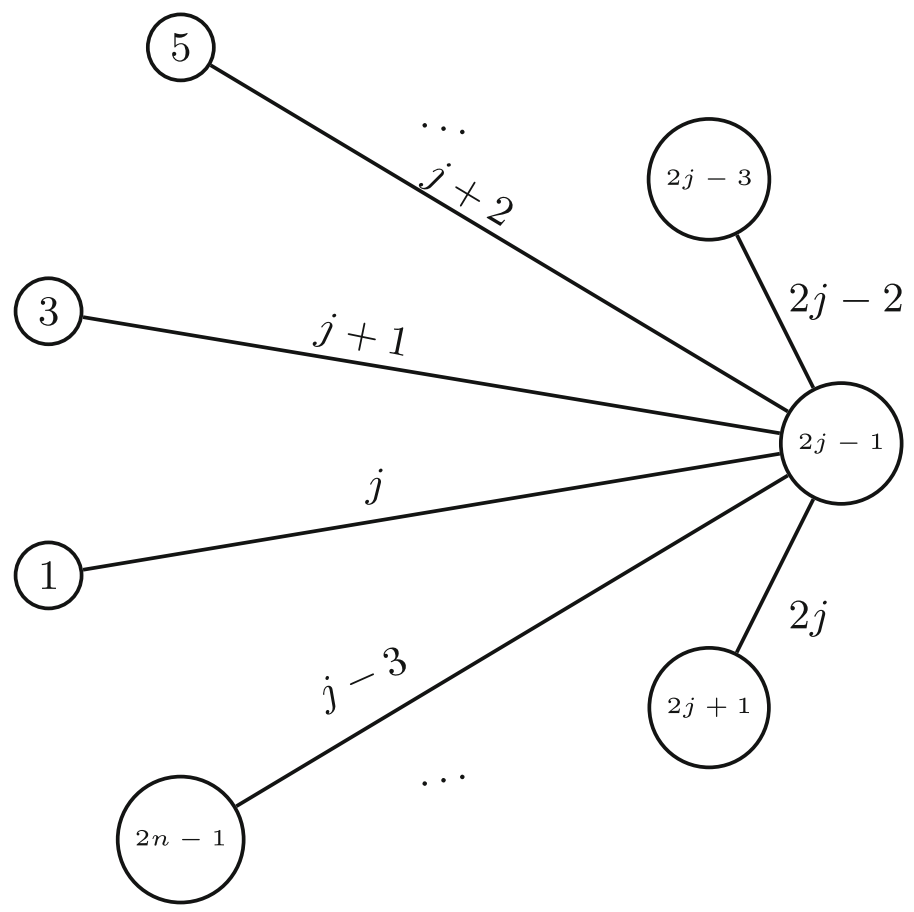

Fig. 3 j-th steps of a total proper coloring of $K_{2 k+1}$ 


\subsection{Bipartite Graphs}

Proposition 5 Let $K_{p, q}$ be a complete bipartite graph of order $p+q$. Then

$$
\operatorname{tndi}_{\Sigma}\left(K_{p, q}\right)= \begin{cases}\Delta+1, & \text { if } p<q \\ \Delta+2, & \text { if } p=q\end{cases}
$$

Proof We can color the edges of $K_{p, q}$ with colors 1, 2, $\cdots, \Delta$ using Konnig's theorem. If $p=q$, then all vertices are of maximum degree, and by Observation 1, we have to use two additional colors: $\Delta+1$ for the first independent set and $\Delta+2$ for the other one. Clearly, this is a proper total coloring distinguishing adjacent vertices by sums.

If $p<q$, we color with $\Delta+1$ all vertices with maximum degree. Now, let $v$ be a vertex with degree $p<\Delta$. We can choose at least one color for $v$ from the set $\{1,2, \cdots, \Delta\}$, which wasn't used for any edge incident to $v$. It is obviously that this is a proper total coloring distinguishing adjacent vertices by sums.

Quite a similiar proof to the case $p=q$ can be done for $k$-regular bipartite graph.

Proposition 6 Let $G$ be a regular bipartite graph. Then $\operatorname{tndi}_{\sum}(G)=\Delta+2$.

Theorem 2 Let $T$ be a tree of order $n$. Then $\operatorname{tndi}_{\Sigma}(T)=\Delta+2$, if there exist two adjacent vertices with maximum degree in $T$. Otherwise $\operatorname{tndi}_{\sum}(T)=\Delta+1$.

Proof We prove the theorem by induction on the order $n$ of the tree $T$. It is easy to verify that the theorem is valid for $n=1,2,3,4$. Suppose $n \geq 5$ and the theorem is valid for any tree with order $n-1$. Now let $T$ be a tree with order $n$ and $P=x \cdots w y z$ be a longest path in $T$. The ends $x$ and $z$ of $P$ are of degree 1 in $T$. Let $T^{\prime}=T-z$.

Case 1. $\Delta\left(T^{\prime}\right)=\Delta(T)-1$.

It is obvious that $y$ is the only vertex in $T$ with maximum degree and $d_{T}(y) \geq 3$. Let $N_{T}(y)=\left\{w, z, z_{1}, z_{2}, \cdots, z_{t}\right\}$, then $d_{T}\left(z_{i}\right)=1, i=1,2, \cdots, t$. By induction hypothesis, $T^{\prime}$ has a total-neighbor-distinguishing coloring by sums using $\Delta\left(T^{\prime}\right)+2=$ $\Delta(T)+1$ colors. Then we color $y z$ with the only color such that the coloring is proper. There are at least two colors which are available for vertex $z$ such that the coloring is proper and at least one of them can distinguish $y$ and $z$ by sums.

Therefore, $T$ has a total-neighbor-distinguishing coloring by sums using $\Delta(T)+1$ colors.

Case 2. $\Delta\left(T^{\prime}\right)=\Delta(T)$ and there are no two adjacent vertices with maximum degree. By induction hypothesis, $T^{\prime}$ has a total-neighbor-distinguishing coloring by sums using $\Delta(T)+1$ colors. If $y$ is a maximum degree in $T$, it is easy to obtain a $\Delta(T)+1$-total-neighbor-distinguishing coloring by sums as Case 1 .

We assume $y$ is not a maximum degree in $T$. Then there are at least two colors which are available for the edge $y z$ such that the final coloring is proper. And at least one of them can distinguish $y$ and $w$ by sums. There are at least two colors which can color $z$ (and $z_{i}$ if necessary), and at least one of them can distinguish $z,\left(z_{i}\right)$ and $y$ by sums.

Therefore, $T$ has a total-neighbor-distinguishing coloring by sums using $\Delta(T)+1$ colors. 
Case 3. $\Delta\left(T^{\prime}\right)=\Delta(T)$ and there are two adjacent vertices in $T$ with maximum degree. By induction hypothesis, $T^{\prime}$ has a total-neighbor-distinguishing coloring by sums using $\Delta(T)+2$ colors.

When at most one of $w$ and $y$ is a maximum degree in $T$, color $y z$ properly such that $f(w) \neq f(y)$. There are at least two colors can be used for $y z$ and at least one of them can distinguish sums $f(w)$ and $f(y)$.

When both $w$ and $y$ are vertices in $T$ with maximum degree. There are at least two colors which can color $y z$ such that the coloring is proper and at least one of them can distinguish $y$ and $w$ by sums.

There are at least two colors can be used for $z$ and at least one of them can distinguish $y$ and $z$. The color of $z_{i}$ can exchange (if necessary) such that the final coloring is proper and $z_{i}$ and $y$ are distinguished by sums in the new coloring.

Theorem 3 If $G=(X, Y ; E)$ is a bipartite graph, then $\operatorname{tndi}_{\sum}(G) \leq \Delta+3$.

Proof We color the edges of $G$ with colors $1,3,4,5, \cdots, \Delta+1$ using König's theorem. Now we will show that we can choose one free color from the set $\{1,2,3, \cdots, \Delta+1\}$ for every vertex of $X$ such that its sum will be odd if $\Delta \equiv 0$ or $1(\bmod 4)$, and even if $\Delta \equiv 2$ or $3(\bmod 4)$. Next we color the vertices of $Y$ with color $\Delta+2$ or $\Delta+3$ such that its sums will be of a different parity than vertices of $X$. Clearly, this is a proper total coloring distinguishing adjacent vertices by sums.

So, let $\Delta=4 k$. We consider a vertex $x$ of $X$ such that $\operatorname{deg}(x)=\Delta$. Note that the sum

$$
\sum_{e: x \in e} c(e)=1+\frac{3+4 k+1}{2}(4 k-1)=2(k+1)(4 k-1)+1
$$

is an odd number. Thus if we put $c(x)=2$, we obtain an odd sum $f(x)$. Moreover, observe that there are $2 k+1$ odd numbers in the set $\{1,3,4,5, \cdots, 4 k+1\}$, hence we can always find a relevant color for any vertex from $X$ in such a way that its sum will be odd.

Now let $\Delta=4 k+2$. Like above, we consider a vertex $x$ from $X$ such that $\operatorname{deg}(x)=$ $\Delta$. Now, the sum

$$
\sum_{e: x \in e} c(e)=1+\frac{3+4 k+3}{2}(4 k+1)=(4 k+1)(2 k+3)+1=(4 k+1) 2 k^{\prime}
$$

is even. Thus, if we put $c(x)=2$ we obtain an even sum $f(x)$. Moreover, there are $2 k+2$ odd numbers in the set $\{1,3,4, \cdots, 4 k+3\}$, hence also in this case, we can always find a relevant color for any vertex from $X$ in such a way that its sum will be even.

The proofs of the remaining two cases are similar to the described above.

\subsection{Graphs with Maximum Degree Three}

Theorem 4 If $G$ is a 3-regular graph, then $\operatorname{tndi}_{\sum}(G) \leq 6$. 
Fig. 4 Case 1 of the proof: there exists a vertex $x$ of degree one in $G$

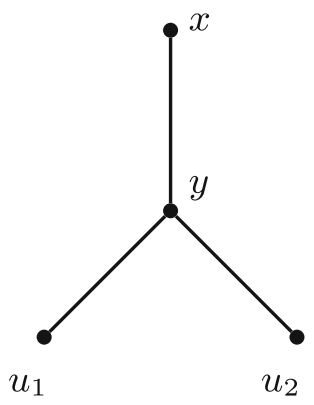

Proof The claim holds for $K_{4}$ by Proposition 4. Thus, $G$ is either biparite or tripartite, by Brook's theorem $(\chi(G) \leq \Delta)$. Hence, by Theorem 3, we can assume that $G$ is a tripartite graph with independent sets $X, Y, Z$. First we color all edges using colors 2, 3, 4, 5-we can do this by Vizing's theorem. Next we can put colors of vertices as follows: $c(x)=1$ for $x \in X, c(y)=6$ for $y \in Y$, and $c(z)$ is a free color from set $\{2,3,4,5\}$ for $z \in Z$. Now we count the sums: $f(x) \in\{10,11,12,13\}, f(y) \in$ $\{15,16,17,18\}$ and $f(z)=14$. So, this is a proper total coloring distinguishing adjacent vertices by sums.

Theorem 5 If $G$ is a graph with $\Delta \leq 3$, then $\operatorname{tndi} \sum_{\sum}(G) \leq 6$.

Proof Throughout this proof we say that a graph has a good coloring $c$ with the function of sums $f$, if $c$ is a proper total coloring of this graph, and for any two adjacent vertices $v$ and $u$ the total sums $f(u)$ and $f(v)$ are different.

We proceed by induction on $n:=|V(G)|$. Observe that the claim is trivial for $n \leq 4$. If all vertices are of degree three in $G$, then a theorem holds by Theorem 4 . So we assume that there exist vertices of degree one or two. We consider three cases.

Case 1. There exists a vertex $x$ of degree one in $G$.

Denote by $y$ the adjacent vertex to $x$ in $G$, and two vertices adjacent to $y$ (if there exists only one, then the proof is the same) by $u_{1}$ and $u_{2}$ (see Fig. 4).

We consider a graph $G^{\prime}=G-x$ and it has a good coloring $c^{\prime}$ with the function of sums $f^{\prime}$ by the induction hypothesis. Now, we define a coloring $c$ of $G$ such that $c(v)=c^{\prime}(v)$ if $v \in V\left(G^{\prime}\right), c(e)=c^{\prime}(e)$ if $e \in E\left(G^{\prime}\right)$. Observe that we have at least one color free for an edge $x y$, because $c(x y)$ have to be different from $c(y), c\left(y u_{1}\right)$, $c\left(y u_{2}\right), f^{\prime}\left(u_{1}\right)-f^{\prime}(y), f^{\prime}\left(u_{2}\right)-f^{\prime}(y)$. Therefore we can choose a color for $x$ such that it will be different from $c(y), c(x y), f^{\prime}(y)$.

Case 2. All vertices are of degree either two or three in $G$, and there exist two adjacent vertices $x_{1}$ and $x_{2}$ of degree two.

If all vertices are of degree two, then $G$ is cycle and a good coloring exists by Proposition 2.

Let $y_{1}$ be a vertex adjacent to $x_{1}$, and let $y_{2}$ be adjacent to $x_{2}$. Observe that vertices $y_{i}$ are of degree two or three, and we can assume that $d\left(y_{1}\right)=3$. First suppose that $y_{1}=y_{2}$, so we have a cycle $C_{3}$ in $G$. Let $y_{1}$ be adjacent to a vertex $u$ (see Fig. 5).

Consider a graph $G^{\prime}=G-\left\{x_{1}, x_{2}\right\}$. Then $G^{\prime}$ has a good coloring $c^{\prime}$ with the function of sums $f^{\prime}$ by the induction hypothesis. Now we define a coloring $c$ of $G$ 
Fig. 5 Case 2 of the proof: there exists a cycle $x_{1} x_{2} y_{1}$ in $G$

Fig. 6 Case 2 of the proof: there exists a path $y_{1} x_{1} x_{2} y_{2}$ in $G$
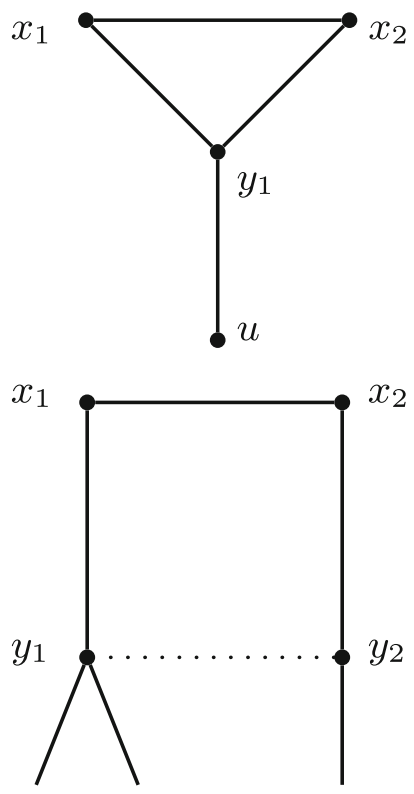

such that $c(v)=c^{\prime}(v)$ if $v \in V\left(G^{\prime}\right)$, and $c(e)=c^{\prime}(e)$ if $e \in E\left(G^{\prime}\right)$. Observe that we can choose two colors $c\left(y_{1} x_{1}\right)$ and $c\left(y_{1} x_{2}\right)$ different from $c\left(y_{1}\right), c\left(y_{1} u\right)$ and such that $c\left(y_{1} x_{1}\right)+c\left(y_{1} x_{2}\right) \neq f^{\prime}(u)-f^{\prime}\left(y_{1}\right)$. Next, we can put $c\left(x_{1} x_{2}\right)=c\left(y_{1}\right)$, and choose two colors $c\left(x_{1}\right)$ and $c\left(x_{2}\right)$ in such a way that

- the color $c\left(x_{1}\right)$ is different from $c\left(y_{1}\right), c\left(y_{1} x_{1}\right)$, and $f\left(x_{1}\right) \neq f\left(y_{1}\right)$;

- the color $c\left(x_{2}\right)$ is different from $c\left(x_{1}\right), c\left(y_{1}\right), c\left(y_{1} x_{2}\right)$, and $f\left(x_{2}\right) \neq f\left(x_{1}\right)$ and $f\left(x_{2}\right) \neq f\left(y_{1}\right)$.

Now, let $y_{1} \neq y_{2}$ and $y_{1} y_{2} \notin E(G)$. We consider a graph $G^{\prime}$ with an additional edge $y_{1} y_{2}$, where $V\left(G^{\prime}\right)=V \backslash\left\{x_{1}, x_{2}\right\}$ (see Fig. 6).

A graph $G^{\prime}$ has a good coloring $c^{\prime}$ with the function of sums $f^{\prime}$ by induction hypothesis. Now we define a coloring $c$ of $G$ such that $c(v)=c^{\prime}(v)$ if $v \in V\left(G^{\prime}\right)$, and $c(e)=c^{\prime}(e)$, if $e \in E\left(G^{\prime}\right) \backslash\left\{y_{1} y_{2}\right\}$. Moreover, let $c\left(x_{1} y_{1}\right)=c\left(x_{2} y_{2}\right)=c^{\prime}\left(y_{1} y_{2}\right)$ and let $c\left(x_{1}\right)=c\left(y_{2}\right)$. Next, if $d\left(y_{2}\right)=2$, then $c\left(x_{1} x_{2}\right)=f^{\prime}\left(y_{2}\right)-c^{\prime}\left(y_{2}\right)-c^{\prime}\left(y_{1} y_{2}\right)$, and we can choose $c\left(x_{2}\right)$ which is different from $c\left(y_{2}\right), c\left(x_{1} x_{2}\right), c\left(x_{2} y_{2}\right)$. Thus, if $d\left(y_{2}\right)=3$ then $c\left(x_{2}\right)=c\left(y_{1}\right)$, and we can choose $c\left(x_{1} x_{2}\right)$ different from $c\left(x_{1}\right), c\left(x_{2}\right)$, $c\left(x_{2} y_{2}\right)$, and such that $f\left(x_{1}\right) \neq f\left(y_{1}\right), f\left(x_{2}\right) \neq f\left(y_{2}\right)$.

Now, let $y_{1} y_{2} \in E$. We consider a graph $G^{\prime}=\left(V^{\prime}, E^{\prime}\right)$, where $V^{\prime}=V \backslash\left\{x_{1}, x_{2}\right\} \cup$ $\{x\}$ and $E^{\prime}=E \backslash\left\{x_{1} y_{1}, x_{2} y_{2}, x_{1} x_{2}\right\} \cup\left\{y_{1} x, y_{2} x\right\}$ (see Fig. 7).

A graph $G^{\prime}$ has a good coloring $c^{\prime}$ with the function of sums $f^{\prime}$, by the induction hypothesis. Now we define a coloring $c$ of $G$ such that $c(v)=c^{\prime}(v)$ if $v \in V\left(G^{\prime}\right)-\{x\}$, and $c(e)=c^{\prime}(e)$ if $e \in E\left(G^{\prime}-x\right)$. Moreover, let $c\left(x_{1} y_{1}\right)=c^{\prime}\left(x y_{1}\right), c\left(x_{2} y_{2}\right)=$ $c^{\prime}\left(x y_{2}\right)$. Next,

1) if $c\left(y_{1}\right) \neq c\left(x_{2} y_{2}\right)$ or $c\left(y_{2}\right) \neq c\left(x_{1} y_{1}\right)$ then [we assume $c\left(y_{1}\right) \neq c\left(x_{2} y_{2}\right)$ ] $c\left(x_{1}\right)=c^{\prime}\left(x y_{2}\right), c\left(x_{1} x_{2}\right)=c^{\prime}(x)$, and we can choose $c\left(x_{2}\right)$ different from $c\left(x_{1}\right)$, $c\left(y_{2}\right), c\left(x_{1} x_{2}\right)$, and such that $f\left(x_{2}\right) \neq f\left(y_{2}\right), f\left(x_{2}\right) \neq f^{\prime}(x)$; 

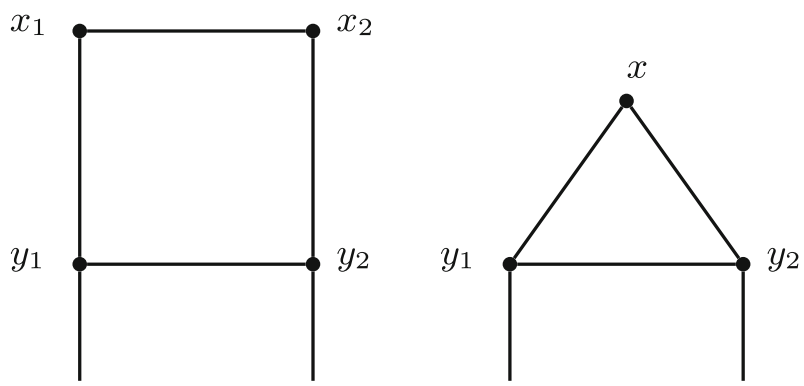

Fig. 7 Case 2 of the proof: there exists a cycle $x_{1} x_{2} y_{1} y_{2}$ in $G$

2) if $c\left(y_{1}\right)=c\left(x_{2} y_{2}\right)$ and $c\left(y_{2}\right)=c\left(x_{1} y_{1}\right)$ then $c\left(x_{1}\right)=a, c\left(x_{2}\right)=b, c\left(x_{1} x_{2}\right)=$ $c^{\prime}\left(y_{1} y_{2}\right)$ [where $a:=f^{\prime}\left(y_{1}\right)-c^{\prime}\left(y_{1}\right)-c^{\prime}\left(y_{1} y_{2}\right)-c^{\prime}\left(x y_{1}\right), b:=f^{\prime}\left(y_{2}\right)-c^{\prime}\left(y_{2}\right)-$ $\left.c^{\prime}\left(y_{1} y_{2}\right)-c^{\prime}\left(x y_{2}\right)\right]$-observe that if it is not possible then we can put either $c\left(x_{1}\right)=\alpha$ or $c\left(x_{2}\right)=\alpha$ (where $\alpha$ is sixth free color different from $\left.c\left(y_{1}\right), c\left(y_{2}\right), c\left(y_{1} y_{2}\right), a, b\right)$ : suppose that in each of the three cases we obtain a conflict, hence

$$
\left\{\begin{array}{l}
c^{\prime}\left(y_{2}\right)+a=c^{\prime}\left(y_{1}\right)+b \\
\alpha=c^{\prime}\left(y_{2}\right)+b \\
\alpha=c^{\prime}\left(y_{1}\right)+a
\end{array}\right.
$$

so

$$
\left\{\begin{array}{l}
c^{\prime}\left(y_{2}\right)+a=c^{\prime}\left(y_{1}\right)+b \\
c^{\prime}\left(y_{2}\right)+b=c^{\prime}\left(y_{1}\right)+a,
\end{array}\right.
$$

what leads to a contradiction (namely $a \neq b$, because $f^{\prime}\left(y_{1}\right) \neq f^{\prime}\left(y_{2}\right)$ ).

Case 3. There exist vertices of degree either two or three in $G$, and every vertex of degree two is adjacent to a vertex of degree three.

Let $x$ be a vertex of degree two and $y_{1}, y_{2}$ be two vertices of degree three adjacent to $x$. We consider a graph $H$, which is created from two copies $G_{1}$ and $G_{2}$ isomorphic to $G$, where every two relevant vertices $x_{1} \in V\left(G_{1}\right)$ and $x_{2} \in V\left(G_{2}\right)$ of degree two are adjacent in $H$ (see Fig. 8).

So a graph $H$ is cubic, and it has a good coloring $c_{H}$, by Theorem 4 . Now we will explain how we can modify $c_{H}\left(x_{1}\right)$ to obtain a good coloring of $G_{1}$, such that $\left.c_{H}\right|_{G_{1}-x_{1}}$ remains unchanged.

First, let $H$ be a bipartite graph with independent sets $A$ and $B$. We can color all edges using colors $2,3,4,5$, by Vizing's theorem, and $c_{H}(a)=1$ for $a \in A$ and $c_{H}(b)=6$ for $b \in B$. Then the sums are different in both sets, because $f_{H}(a) \in$ $\{10,11,12,13\}$ and $f_{H}(b) \in\{15,16,17,18\}$. Observe that if $x_{1} \in A$ then we can delete an edge $x_{1} x_{2}$ and we will still have a proper total coloring distinguishing adjacent vertices by sums. Now let $x_{1} \in B$. If we delate an edge $x_{1} x_{2}$, then we can obtain a conflict: $f_{G}\left(x_{1}\right)=f_{G}\left(y_{1}\right)$ or $f_{G}\left(x_{1}\right)=f_{G}\left(y_{2}\right)$. Then we can choose a good color for $x_{1}$ from the set $\{2,3,4,5\} \backslash\left\{c_{H}\left(x_{1} y_{1}\right), c_{H}\left(x_{1} y_{2}\right)\right\}$.

Hence let $H$ be a tripartite graph, and let $c_{H}$ be defined as in the proof of Theorem 4 . Observe that if $c_{H}\left(y_{1}\right)=c_{H}\left(y_{2}\right)=6$ then $f_{H}\left(x_{1}\right) \in\{10, \cdots, 14\}, f_{H}\left(y_{1}\right)$ and 


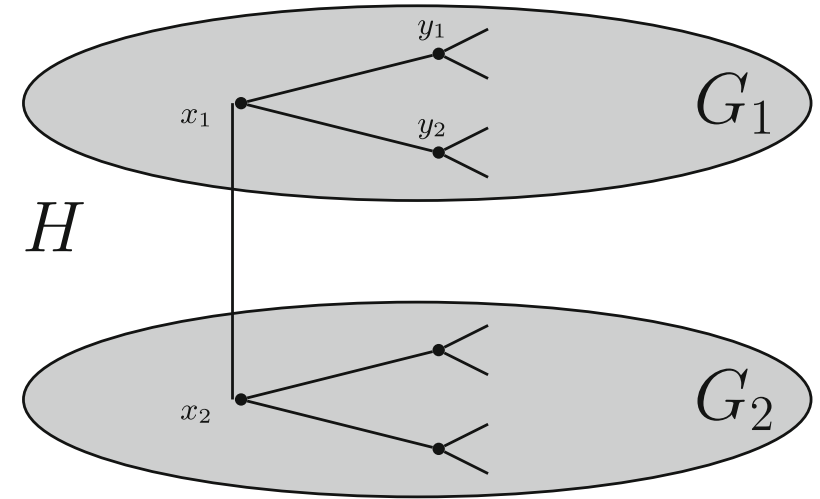

Fig. 8 Case 3 of the proof: a construction of graph $H$ from two copies of $G$

$f_{H}\left(y_{2}\right) \in\{15, \cdots, 18\}$. So, we can delete an edge $x_{1} x_{2}$ (its color is from the set $\{2,3,4,5\})$, and we will still have a good coloring of $G_{1}$.

If $c_{H}\left(y_{1}\right)=c_{H}\left(y_{2}\right)=1$, then we have to choose a color $c_{G}\left(x_{1}\right)$ from the set $\{2,3,4,5,6\} \backslash\left\{c_{H}\left(x_{1} y_{1}\right), c_{H}\left(x_{1} y_{2}\right)\right\}$ such that $f_{G}\left(x_{1}\right) \neq f_{H}\left(y_{1}\right)$ and $f_{G}\left(x_{1}\right) \neq$ $f_{H}\left(y_{2}\right)$.

If $f_{H}\left(y_{1}\right)=f_{H}\left(y_{2}\right)=14$ then if the color of $x_{1}$ is not 1 , then recolor $x_{1}$ by 1 , so and $f_{G}\left(x_{1}\right) \in\{6, \cdots, 10\}$. If $c_{H}\left(y_{1}\right)=6$ and $f_{H}\left(y_{2}\right)=14$ then we delete $x_{1} x_{2}$ and we also put $c_{G}\left(x_{1}\right)=1, f_{G}\left(x_{1}\right) \in\{6, \cdots, 10\}$, while $f_{H}\left(y_{1}\right) \in\{15, \cdots, 18\}$.

If $c_{H}\left(y_{1}\right)=1$ and $c_{H}\left(y_{2}\right)=6$ then for $c_{G}\left(x_{1}\right)$ we can choose $c_{H}\left(x_{1}\right)$ or $c_{H}\left(x_{1} x_{2}\right)$ such that $f_{G}\left(x_{1}\right) \neq f_{H}\left(y_{1}\right)$ (we know that $f_{G}\left(x_{1}\right) \neq f_{H}\left(y_{2}\right)$, because $f_{G}\left(x_{1}\right) \leq 12$ and $\left.f_{H}\left(y_{2}\right) \geq 15\right)$.

The last subcase is if $c_{H}\left(y_{1}\right)=1$ and $f_{H}\left(y_{2}\right)=14$. We have to choose a color $c_{G}\left(x_{1}\right)$ from the set $\{2,3,4,5,6\} \backslash\left\{c_{H}\left(x_{1} y_{1}\right), c_{H}\left(x_{1} y_{2}\right), c_{H}\left(y_{2}\right)\right\}$ such that $f_{G}\left(x_{1}\right) \neq$ $f_{H}\left(y_{1}\right)$ and $f_{G}\left(x_{1}\right) \neq f_{H}\left(y_{2}\right)$. If we have both conflicts and we cannot choose a color $c_{G}\left(x_{1}\right)$, then $c_{H}\left(x_{1} y_{1}\right) \neq c_{H}\left(y_{2}\right)$.

Let $c_{G}\left(x_{1} y_{1}\right)=\alpha, c_{G}\left(x_{1} y_{2}\right)=\beta$ and $c_{G}\left(y_{2}\right)=\gamma$, then $\alpha, \beta, \gamma \in\{2,3,4,5\}$ and they are pairwise distinct.

a) $f_{G}\left(x_{1}\right)=f_{G}\left(y_{2}\right)$, i.e., $\alpha+\beta+6=14$, then $\alpha+\beta=8$. Therefore, $\left\{\begin{array}{l}\alpha=3 \\ \beta=5\end{array}\right.$ or $\left\{\begin{array}{l}\alpha=5 \\ \beta=3\end{array}\right.$. Since the neighbors of $y_{2}$ are colored by either 1 or 6 , exchange $\beta$ with $\gamma$, the the sum of colors of $y_{2}$ is 14 . The vertices $x_{1}$ and $y_{2}$ can be distinguished by sums in the new coloring. If $x_{1}$ and $y_{2}$ are also distinguished, it is a good coloring. Otherwise, $\alpha+$ $\gamma \in\{5,6,7\}$ in the new coloring. So, $\left\{\begin{array}{l}\alpha=3 \\ \beta=5 \\ \gamma=2\end{array}\right.$ or $\left\{\begin{array}{l}\alpha=3 \\ \beta=5 \\ \gamma=4\end{array}\right.$ or $\left\{\begin{array}{l}\alpha=5 \\ \beta=3 \\ \gamma=2\end{array}\right.$. If $\left\{\begin{array}{l}\alpha=3 \\ \beta=5 \\ \gamma=2\end{array}\right.$, then we recolor $x_{1}$ with 4 . The new coloring is a good coloring. The remaining two cases are analogous.

b) $f_{G}\left(x_{1}\right)=f_{G}\left(y_{1}\right)$, i.e., $\alpha+\beta+6 \in\{11,12,13\}$, then $\alpha+\beta \in\{5,6,7\}$. Since the neighbors of $y_{2}$ are colored by either 1 or 6 , exchange $\beta$ with $\gamma$, then the vertices $x_{1}$ and $y_{2}$ can be distinguished by sums and the the sum of colors of $y_{2}$ is 14 in the 
new coloring. If $x_{1}$ and $y_{2}$ are also distinguished, it is a good coloring. Otherwise, $\alpha+\gamma+6=14$ in the new coloring. Therefore, $\left\{\begin{array}{l}\alpha=3 \\ \beta=5\end{array}\right.$ or $\left\{\begin{array}{l}\alpha=5 \\ \beta=3\end{array}\right.$. So, $\left\{\begin{array}{l}\alpha=3 \\ \beta=2 \\ \gamma=5\end{array}\right.$ or $\left\{\begin{array}{l}\alpha=3 \\ \beta=4 \\ \gamma=5\end{array}\right.$ or $\left\{\begin{array}{l}\alpha=5 \\ \beta=2 \\ \gamma=3\end{array}\right.$. Analogous to Case a), we can have a good coloring.

Open Access This article is distributed under the terms of the Creative Commons Attribution License which permits any use, distribution, and reproduction in any medium, provided the original author(s) and the source are credited.

\section{References}

1. Bondy, J.A., Murty, U.S.R.: Graph theory with applications. Macmillan, London (1976)

2. Chen, $X .:$ On the adjacent vertex distinguishing total coloring numbers of graphs with $\Delta=3$. Discret. Math. 308-17, 4003-4007 (2008)

3. Flandrin, E., Marczyk, A., Przybyło, J., Saclé, J.F., Woźniak, M.: Neighbor sum distinguishing index. Graphs Comb. 29, 1329-1336 (2013)

4. Hulgan, J.: Concise proofs for adjacent vertex-distinguishing total coloring. Discret. Math. 309, 25482550 (2009)

5. Kostochka, A.V: The total coloring of a multigraph with maximal degree 4. Discret. Math. 17-2, 161163 (1977)

6. Kostochka, A.V.: Upper bounds of chromatic functions of graph (in Russian). Ph.D. Thesis, Novosibirsk (1978)

7. Kalkowski, M., Karoński, M., Pfender, F.: Vertex-coloring edge-weightings: towards 1-2-3conjecture. J. Combin. Theory Ser. B 100-3, 347-349 (2010)

8. Karoński, M., Łuczak, T., Thomason, A.: Edge veights and vertex colours. J. Combin. Theory Ser. B 91, 151-157 (2004)

9. Przybyło, J., Woźniak, M.: On a 1,2 Conjecture. Discret. Math. Theor. Comput. Sci. 12, 101-108 (2010)

10. Rosenfeld, M.: On the total coloring of certain graphs. Israel J. Math. 9-3, 396-402 (1970)

11. Zhang, Z., Chen, X., Li, J., Yao, B., Lu, X., Wang, J.: On adjacent-vertex-distinguishing total coloring of graphs. Sci. China Ser. A Math. 48-3, 289-299 (2005) 